JOURNAL OF THE

CHUNGCHEONG MATHEMATICAL SOCIETY

Volume 26, No. 3, August 2013

\title{
SPHERICAL CAPS IN A CONVEX CONE
}

\author{
TAEKWAN UM*
}

\begin{abstract}
We show that a compact embedded hypersurface with constant ratio of mean curvature functions in a convex cone $C \subset$ $\mathbb{R}^{n+1}$ is part of a hypersphere if it has a point where all the principal curvatures are positive and if it is perpendicular to $\partial C$.
\end{abstract}

\section{Introduction}

Let $S$ be a hypersurface in the $n+1$ dimensional Euclidean space $\mathbb{R}^{n+1}$. Its $r$ th mean curvature function $H_{r}$ is the $r$ th elementary symmetric function of principal curvature function of $M$ divided by $\left(\begin{array}{c}n \\ r\end{array}\right)$. Hence the Gauss-Kronecker curvature is $H_{n}$ and the usual mean curvature function is $H_{1} . H_{0}$ is defined to be one. It is well known that an embedded closed hypersurface in $\mathbb{R}^{n+1}$ with nonzero constant mean curvature function $H_{r}$ is a round sphere $[1,6]$. A closed embedded hypersurface in $\mathbb{R}^{n+1}$ with constant ratio of mean curvature functions, $H_{k} / H_{r}=c$, is also a round sphere $[4,5]$.

Among embedded compact hypersurfaces with nonempty boundary, it is known that compact embedded hypersurface in $\mathbb{R}^{n+1}$ with nonzero constant $H_{r}, r \geq 2$ and spherical boundary are spherical caps, that is, part of a round hypersphere [2]. It is also known recently in [3] that a compact embedded hypersurface with constant $H_{r}$ in a convex piecewise smooth cone $C$ which is perpendicular to $\partial C$ is part of a spherical cap. In this paper, we generalize this in the following theorem:

Theorem 1.1. Let $C$ be a domain in $\mathbb{R}^{n+1}$ which is a convex cone with piecewise smooth boundary $\partial C$ and with the vertex at the origin. Let $S \subset C$ be an embedded compact hypersurface with boundary in $\partial C$ such that $S$ is perpendicular to $\partial C$ along $\partial S$. If there is a point on $S$ where all the principal curvatures are positive and if the ratio $H_{k} / H_{l}$ is

Received May 09, 2013; Accepted July 19, 2013.

2010 Mathematics Subject Classification: Primary 53A35.

Key words and phrases: spherical caps, mean curvature functions. 
a nonzero constant for some $k, l=1,2, \cdots, n, k \neq l, S$ is a spherical cap.

\section{Proof}

Let $C$ be a domain in $\mathbb{R}^{n+1}$ which is a convex cone with piecewise smooth boundary $\partial C$ and with the vertex at the origin. Let $S \subset C$ be an embedded compact hypersurface with boundary in $\partial C$ such that $S$ is perpendicular to $\partial C$ along $\partial S$. Let $\eta$ be the unit normal vector field of the embedding $X: S \rightarrow \mathbb{R}^{n+1}$.

The following Lemma is given in [3].

Lemma 2.1. The following holds for $k=1,2, \cdots, n$ :

$$
\int_{S}\left(H_{k-1}-H_{k}\langle X, \eta\rangle\right)=0 .
$$

The following lemma is given in [4].

Lemma 2.2. Suppose $H_{k}>0$ for some $k \geq 2$. Then the followings hold:

(i) For any $j=1,2, \cdots, k, H_{j}>0$. Moreover, $H_{k}^{\frac{k-1}{k}} \leq H_{k-1}$.

(ii) $H_{k} / H_{k-1} \leq H_{k-1} / H_{k-2}$.

(iii) For every $l<k, H_{k} / H_{l} \leq H_{k-1} / H_{l-1}$.

Now, since $S$ is compact, one can find a point in $S$ where all the principal curvatures are positive. Without loss of generality, we may assume that $1 \leq l<k \leq n$. Then all $H_{k}$ 's are positive at that point. Since $H_{k} / H_{l}$ is constant on $S$ and since $H_{l}$ does not vanish on $S$ by assumption, $H_{k}$ and $H_{l}$ are both positive on $S$. Then from Lemma 2.2 (iii), we have

$$
0<\alpha:=H_{k} / H_{l} \leq H_{k-1} / H_{l-1} .
$$

Since $H_{k}=\alpha H_{l}$, we have by Lemma 2.1

$$
\begin{aligned}
0 & =\int_{S}\left(H_{k-1}-H_{k}\langle X, \eta\rangle\right) \\
& =\int_{S}\left(H_{k-1}-\alpha H_{l}\langle X, \eta\rangle\right),
\end{aligned}
$$

that is, we have

$$
\int_{S} H_{k-1}=\int_{S} \alpha H_{l}\langle X, \eta\rangle
$$


On the other hand, since $\alpha$ is constant, we also have by Lemma 2.1

$$
\int_{S} \alpha\left(H_{l-1}-H_{l}\langle X, \eta\rangle\right)=0
$$

that is, we have

$$
\int_{S} \alpha H_{l-1}=\int_{S} \alpha H_{l}\langle X, \eta\rangle .
$$

From (2.2) and (2.3), we have

$$
\int_{S}\left(H_{k-1}-\alpha H_{l-1}\right)=0 .
$$

Since we have from (2.1) and Lemma 2.2 (i), $H_{k-1}-\alpha H_{l-1} \geq 0$, it follows that

$$
H_{k-1} / H_{l-1}=\alpha=H_{k} / H_{l}
$$

everywhere on $S$. Now proceeding inductively, we have finally

$$
H_{k-l}=H_{k-l} / H_{0}=\alpha
$$

everywhere on $S$. Thus by the aforementioned result of [3], $S$ is a spherical cap.

\section{References}

[1] A. D. Alexandrov, A characteristic property of spheres, Ann. Mat. Pura Appl. 58 (1962), 303-315.

[2] L. J. Alias and J. A. Malacarne, Constant scalar curvature hypersurfaces with spherical boundary in Euclidean space, Rev. Mat. Iberoamericana 18 (2002), 431-442.

[3] J. Choe and S. H. Park, Capillary surfaces in a convex cone, Math. Zeit. 267 (2011), 875-886.

[4] S. E. Koh, Sphere theorem by means of the ratio of mean curvature functions, Glasgow Math. J. 42 (2000), 91-95.

[5] S. E. Koh and S. W. Lee, Addendum to the paper: Sphere theorem by means of the ratio of mean curvature functions, Glasgow Math. J. 43 (2001), 275-276.

[6] N. J. Korevaar, Shere theorem via Alexandrov for constant Weingarten hypersurfaces: Appendix to a note of A. Ros, Jour. Diff. Geom. 27 (1988), 221-223.

*

Department of Mathematics

Konkuk University

Seoul 143-701, Republic of Korea 